\title{
Interleukin-12 and Interleukin-4 Levels of Multibacillary Leprosy Patients Before and After Rifampicine Ofloxacine Minocycline Combined Therapy
}

\author{
Suci Budhiani*, Sri Vitayani Muchtar, Safruddin Amin \\ Department of Dermatology and Venereology, Medical Faculty, Hasanuddin University, Makassar, Indonesia \\ Email address: \\ oetjy_doc@yahoo.com (S. Budhiani) \\ ${ }^{*}$ Corresponding author
}

To cite this article:

Suci Budhiani, Sri Vitayani Muchtar, Safruddin Amin. Interleukin-12 and Interleukin-4 Levels of Multibacillary Leprosy Patients Before and After Rifampicine Ofloxacine Minocycline Combined Therapy. American Journal of Clinical and Experimental Medicine.

Vol. 5, No. 4, 2017, pp. 134-137. doi: 10.11648/j.ajcem.20170504.15

Received: February 18, 2017; Accepted: March 6, 2017; Published: July 3, 2017

\begin{abstract}
Leprosy is a chronic infectious disease caused by Mycobacterium leprae, which is an obligate intracellular bacteria. This study aims to determine the interleukin-12 and interleukin-4 levels of multibacillary leprosy patients before and after Rifampicine Ofloxacine Minocycline (ROM) combined therapy for three months. The research used an analytic observational design with the prospective cohort study pre and post treatment method. The samples were taken at the polyclinic of Skin Treatment Central Public hospital and Ibnu Sina hospital. Samples examination was conducted in the NECHRI laboratory, Hasanuddin University Teaching hospital. The samples were ten new patients of MB type leprosy. Blood samples were taken before and after the ROM therapy for three months. The levels of interleukin-12 and interleukin- 4 were assessed using the ELISA technique. The results shows that there is no significant change in the levels of interleukin-12 and interleukin-4 in the patients of multibacillary leprosy before and after ROM therapy for three months.
\end{abstract}

Keywords: Interleukin-12, Interleukin-4, MB Type Leprosy, ROM

\section{Introduction}

Leprosy is a chronic infectious disease caused by the bacterium Mycobacterium Leprae, an obligate intracellular bacteria. The disease is characterized by slow progressivity, high infectivity and low pathogenicity. Dermatoneurologic signs and symptoms are the main manifestations, ranging in spectrum between two stable poles (tuberculoid and lepromatous). Operational classification of leprosy was made for therapeutic purposes, divided the patients into two groups, namely pausibacillary and multibacillary. Transmission among humans is thought to be the main way of dissemination. The upper respiratory tract of multibacillary patients (lepromatous and borderline) is a key outlet of the bacterium, which then infect others through their upper respiratory tract, especially the nasal mucosa by contaminated droplets [1]. The suspected main source of infection is multibacillary patients, who carry a lot of bacteria on their skin, and capable of transmitting large amounts of bacteria from the nose, with an average of $10^{7}$ per day [2]. The importance role of the immune system in leprosy is indicated by the clinical manifestations and determined by the host immune system. Host produce both humoral and cellular immunity against $\mathrm{M}$. Leprae but it shows opposite correlation across the spectrum of leprosy and is associated with the number of bacilli [3].

Multibacillary leprosy (Lepromatous) is characterized by low cellular immunity with humoral Th2 response [4], as indicated by the production of transforming growth factor (TGF) $-\beta$, IL-4, IL-5 and IL-10 and high antibody production. Instead, pausibacillary patients show high cellular immunity, characterized by Th1 response with the production of IFN- $\gamma$, IL-2, IL-7, IL-12, IL-15 and IL-18 [5]. In multibacillary leprosy, there is an inhibition of IL-12 signal and stimulation response of IL-4, thus these patients are unable to limit M. Leprae's growth [6].

MDT therapy has been proven effective in the treatment and control of leprosy. However, the lack of compliance (associated with the long duration and socioeconomic 
factors), drug resistance and relapse have been reported, which encourage prompt efforts to establish a new treatment regimens to shorten duration of therapy and increase patient compliance while maintaining and enhancing the therapeutic benefits from previous regimens [7].

One alternative regimen recommended by WHO since 1998 is the provision of single dose combination of rifampicine $(600 \mathrm{mg})$, ofloxacine $(400 \mathrm{mg})$, and minocycline (100 mg) (ROM)for PB type leprosy patients with single lesion $[7,8]$. A study in Brazil also found that patients treated with MDT MB compared to ROM for 24 months showed the same results in terms of reductions in Bacterial Index (BI), and improvement in clinical and histopathological features [9]. However, a meta-analysis study comparing multiple doses of ROM to MDT in MB leprosy patients, did not provide sufficient data to conclude the efficacy of ROM therapy in $\mathrm{MB}$ patients. Thus additional study is required to evaluate effectiveness of this regimen in MB leprosy. [7].

Of the few studies available, it appears that MDT-MB and ROM provide the same effectiveness in clinical, bacteriological and histopathological improvement. But to the author's knowledge there has been no study comparing the treatment effect of MDT-MB and ROM in terms of cytokine profile while known from the above description that cytokine profile of leprosy patients greatly affect the spectrum of its clinical manifestations. There has been one study analyzing the effect of MDT-MB therapy on the levels of cytokines IL-12 and IL-4 representing Th1 and Th2 immune responses consecutively after 3 months. Therefore, we conducted an analysis study of IL-12 and IL-4 levels, after 3 months treatment ROM therapy on MB leprosy patients. To monitor and assess the results of the analysis, we included selected patients who meet the criteria as multibacillary samples according to WHO criteria, and measurement of levels IL-12 and IL-4 performed before treatment and after 3 months of therapy with the ROM regimen.

\section{Materials and Methods}

\subsection{Study Place and Period}

The study was carried out at the polyclinic of Skin Treatment Central Public Hospital, and Ibnu Sina Hospital where the samples were collected, and NECHRI laboratory of Hasanuddin University Hospital for cytokine measurement using ELISA technique, from April 2016 - July 2016.

\subsection{Study Design and Variable}

This is an analytic observational study with prospective cohort pre- and post treatment method. Study variables consist of: independent variable (M. Leprae), in-between variable (immunity response), controlled variable (ROM, age), and dependent variable (IL-12, IL-4).

\subsection{Population and Sample}

Study population were newly diagnosed MB leprosy patients (WHO classification) and visited dermatovenereology polyclinic general central hospital Dr.
Wahidin Sudirohusodo Makassar, Skin Treatment Central Public Hospital, Tajuddin Khalid hospital, Ibnu Sina Hospital and other network hospital. Study samples were all population who met the inclusion criteria. Samples were taken consecutively, with total number were 10 patients.

\subsection{Data Collection}

All eligible patients were recorded for the complete identity (name, registration number, age, gender, address), medical history and physical examination to establish the diagnose of MB leprosy. Before treatment photograph were taken and patients were asked to read and signed informed consent. Blood sample were taken twice, first before treatment and second after 3 months treatment with ROM to determine IL-12 and IL-4 levels using ELISA technique.

\subsection{Data Analyze}

Data were processed using Statistical Package for Social Science (SPSS) ver. 23 and presented in the form of table or graphic. Statistical analysis was performed using Wilcoxon test with significance value of $\mathrm{p}<0,05$.

\section{Result}

Study on IL-12 and IL-4 levels of MB leprosy patients before and after 3 months treatment with combination of Rifampicine Ofloxacine and Minocycline was done in analytical observational manner using cohort prospective method. This study was conducted at Skin Treatment Centre Public hospital and Ibnu Sina hospital Makassar, from April 2016 - July 2016. The samples were examined at NECHRI laboratory of Hasanuddin University Hospital using ELISA technique.

Samples were ten MB type leprosy patients whose blood were taken twice, before treatment and after three months treatment with ROM. The youngest patient was 17 year old and the eldest was 57 year old. All samples were examined to determine and analyze the levels of IL-12 and IL-4 using ELISA technique. Coincidentally, all patients in this study were male $(100 \%)$. Table 1 showed characteristic of sample according to sex.

Table 1. Respondent Characteristic according to Sex

\begin{tabular}{lll}
\hline Sex & Number & Percentage (\%) \\
\hline Male & 10 & 100 \\
Female & 0 & 0 \\
Total & 10 & 100 \\
\hline
\end{tabular}

Patients distribution according to age showed that majority were in the 46-60 years of age (40\%), while patients of 18-25 years and 26-45 years both had the same number $(30 \%)$.

Table 2. Respondent Characteristic according to Age.

\begin{tabular}{lll}
\hline Age & Number & Percentage (\%) \\
\hline $18-25$ & 3 & 30 \\
$26-45$ & 3 & 30 \\
$46-60$ & 4 & 40 \\
Total & 10 & 100 \\
\hline
\end{tabular}


Acid fast bacillary (AFB) count before and after three months treatment with ROM showed that from ten samples, majority ( 5 patients) had $+1,3$ patients had +2 , and only 1 patients had +3 . While after treatment, there were 3 patients with $\mathrm{AFB}$ count $+1,2$ patients had +2 and 3 patients had negative results. There was one sample that was not examined for AFB count before treatment and another one sample did not undergo AFB count after treatment.

Table 3. Acid Fast Bacilli Count Before and After 3 Months Treatment with ROM.

\begin{tabular}{lll}
\hline \multirow{2}{*}{ AFB } & Sample Number & \\
\cline { 2 - 3 } & Before & After \\
\hline+1 & 5 & 3 \\
+2 & 3 & 2 \\
+3 & 1 & 0 \\
Negative & - & 3 \\
\hline
\end{tabular}

Comparison of IL-4 level before and after three months treatment with ROM showed that there were no significant difference in the index means of IL- 4 before and after treatment with $\mathrm{p}=0,721$, thus $\mathrm{p}>0,05$.

Table 4. Comparison of IL-4 Level Before and After 3 Months Treatment with ROM.

\begin{tabular}{lll}
\hline & IL-4 $(\mathbf{p g} / \mathbf{m l})$ & \\
\cline { 2 - 3 } & Before & After \\
\hline Mean & 27,86 & 21,15 \\
Median & 18,71 & 13,67 \\
Standard deviation & 32,43 & 24,92 \\
Range & $5,05-115,90$ & $5,63-89,02$ \\
\hline
\end{tabular}

Wilcoxon test $\mathrm{p}=0,721$

Comparison of IL-12 level before and after three months treatment with ROM showed that there were no significant difference in the index means of IL-12 before and after treatment with $\mathrm{p}=0,678$, thus $\mathrm{p}>0,05$.

Table 5. Comparison of IL-12 Level Before and After 3 Months Treatment with ROM.

\begin{tabular}{lll}
\hline & IL-12 (pg/ml) & \\
\cline { 2 - 3 } & Before & After \\
\hline Mean & 390,72 & 315,35 \\
Median & 289,28 & 296,49 \\
Standard deviation & 311,14 & 105,83 \\
Range & $133,25-1210,8$ & $204,41-572,51$ \\
\hline
\end{tabular}

Wilcoxon Test $\mathrm{p}=0,678$

\section{Discussion}

The results from this study showed that the level of IL-12 and IL-4 before and after three months treatment with ROM not differ siginificantly. Number of patients were ten which were all male $(100 \%)$. From the literature,male had higher prevalence of MB type leprosy. This might be caused of male are more prone to MB type leprosy compare to female, thus there were more male patients visit dermatology clinic or hospital to seek for treatment [1].

In this study, most patients (40\%) were in $46-60$ years group of age. One study from Paraiba Federal University showed that leprosy had highest frequency at older age, 5064 year $(27 \%)$. The high incidence in this group of age might be caused by the long incubation period of leprosy, with mean duration of 2-7 years [1]. Another explanation on this phenomena is that leprosy occured in older population who lived in endemic area were frequently caused by reinfection or superinfection from people who had had subclinical leprosy in the past, however, they underwent diminished immune response to leprosy as they aged [10].

Acid fast bacillary count in this study range from +1 to +3 , which showed that these patients were in the form of borderline leprosy, who had unstable immune system. Leprosy spectrum consists of steady chronic pole and borderline form. At the tuberculoid pole (TT) the cellullar immune response is pronounce and stable, and shows low level of antibodies, while in lepromatous pole (LL) shows abundant antibodies yet low cellular immune response. Between these two stable poles, there are borderline form with unstable immune status, that is $\mathrm{BT}, \mathrm{BB}$ and $\mathrm{BL}$ [11].

Borderline leprosy $(\mathrm{BB})$ represents the peak of unstable immunity. The balance between bacillary multiplication and cellular immunity is so tenous and are seldom settle in without one surpassing another. In BT form, clinical manifestation is the result of compound intense cellular infiltration and unstable immunity, which may improve to TT form or deteriorate to BL. BL type leprosy represents peak of expeditious immunity deterioration, consist of compound of immunity imbalance and bacillary multiplication. It has been reported that borderline form were more frequently encountered than polar form [12].

In association with cytokine profile in leprosy patients, it has been known that the two pole of leprosy spectrum were being represented by different $\mathrm{T}$ cell response and cytokine production. In TT pole, patient shows intense cellular immunity to M. Leprae which effectively restrict bacterial growth through production of local Th-1 cytokine, namely IL12 and IFN- $\gamma$. On the other side of pole (LL) characterized by widespread multibacillary disease and lack response to $\mathrm{M}$. leprae, activation of IL-2 which inhibit Th-1 cell, resulting in defective cellular immunity to M. leprae [13, 14].

Subject in this study were patients diagnosed with $\mathrm{MB}$ type leprosy. It is characterized by low cell-mediated immunity with humoral response Th-2, and mRNA produced mainly IL-4, IL-5 and IL-10. These cytokine were presumed to contribute to immunity unresponsiveness and failure activation of macrophage in lepromatous leprosy, hence it is unable to resist bacterial growth [6].

Cells from immune system produce cytokine as response to antigenic stimulation. In this case, cytokine of leprosy patients produced as response to immune stimulation by $\mathrm{M}$. leprae antigen, and when this antigen reduced after treatment, cytokine production also decreased and it may reduced to normal level as in healthy subject if this antigen is totally diminished [15].

Interleukin-4 was first known as important cytokine in the development of Th-2 response, while IL-12 represents Th-1 
response. In this study, there were no siginificant difference in both IL-4 or IL-12 levels before and after treatment with ROM for three months. This result is in accordance with study by Bandjar (2015) who investigate the effect of MDT-MB treatment after three months on IL-12 and IL-4 levels [16]. These results was potentially due to some influential factors.

Firstly, leprosy is a disease with unstable immune system. At the tuberculoid (TT) pole it shows intense cellular immunity with low antibody titer, while lepromatous (LL) pole is characterized by poor cellular immunity along with increased anitbody titer. Among these two stable pole there are borderline form with extremely unstable immunologic status [11]. In this study, the AFB count range from +1 to +3 which shown that the patients in this study were in the form of borderline leprosy whom immune status were considerably unstable.

Secondly, eventually the patients in this study had mixed cytokine pattern Th0, due to none of them were in the stable form. A study showed that half patients from various leprosy spectrum showed mixed cytokine pattern, despite in the polar form. Hetereogenity in Th phenotype, that is some patients had Th0 whereas others with same clinical type had Th1 or Th2 response, most likely associated with time kinetic of dynamic process involved in $\mathrm{T}$ cell naive differentiation to polar phenotype [17].

Thirdly, the short follow up duration after treatment in this study which is only three months. Study by Madan et al (2011) reported decreasing in level of all examined cytokine serum (TNF- $\alpha$, IFN- $\gamma$, IL-1 $\beta$ and IL-10) in PB type leprosy patients after treatment with MDT for six months and in MB type (except IFN- $\gamma$ ) after MDT treatment for 1 year [11].

Limitations of this study including short after treatment follow up duration, no control, no histopathological classification, and no screening for malnutrition and disease such as parasite infestation, allergic rhinitis, asthma bronchiale and atopic dermatitis, hence the influence of these condition to the increasing or decreasing level of cytokine can not be avoided.

As far as the researcher awareness, this study is the first to evaluate the effect of ROM therapy on cytokine profile of MB leprosy patients. However, due to its limitations, the results is yet to be representative of ROM effectivity. Thus further studies with more rigorous screening are needed.

\section{Conclusion and Suggestion}

Researcher concluded that the levels of IL-12 and IL-4 were not significantly difference after treatment with ROM for three months. These might be due to factors include the immune status of patients were unstable and had mixed cytokine pattern as they are in borderline form, and short follow up duration, which was only three months.

It is suggested to perform extended study to evaluate level of IL-12 and IL-4 gradually after 6, 9 and 12 months treatment of $\mathrm{MB}$ leprosy patients. More rigorous sample screening should be done to diminish all the limitations of this study that will be expected to yield more accurate results.

\section{References}

[1] Correa et al. (2012). Epidemiological, clinical, and operational aspects of leprosy patients assisted at a referral service in the state of Maranhao, Brazil. Rev Soc Bras Med Trop 45 (1): 89-94.

[2] Martins et al. (2012). Pathogen-Specific Epitopes as Epidemiological Tools For Defining The Magnitude of Mycobacterium Leprae Transmission in Areas Endemic for Leprosy. PloSNegl Trop Dis 6(4): e1616.

[3] Parkash.(2009). Classification of Leprosy IntoMultibacillary and Paucibacillary Groups: An Analysis. FEMS Immunol Med Microbiol 55: 1-5.

[4] Walker \&Lockwood. (2006). The Clinical and Immunological Features of Leprosy. British Medical Bulletin 77 and 78: 103-21.

[5] Jarduli et al. (2013). Role of HLA, KIR, MICA and Cytokines Genes in Leprosy. Hindawi Publishing Corporation. 1-17.

[6] Modlin RL. 1994. Th1-Th2 paradigm: insight from leprosy. J Invest Dermatol 102: 828.

[7] Setia et al. (2011). Is There a Role for Rifampicin, Ofloxacin, and Minocycline (ROM) Therapy in the Treatment of Leprosy? Systematic Review and Meta-Analysis. Trop Med \& Intern Health.16: 1541-51.

[8] Worobec. (2012). Current Approaches and Future Directions in The Treatment of Leprosy. Research and Reports in Trop Med; 3: 79-91.

[9] Lockwood \&Cunha.(2012). Developing New MDT Regimens for MB Patients: Time to Test ROM 12 Month Regimens Globally. Lepr Rev 83: 241-4.

[10] Noordeen, S. K. (1994) Epidemiology of leprosy. In: Hastings, R. C. \& Opromolla, D. V. A. (Eds.) Leprosy. $2^{\text {nd }}$ ed. Edinburgh, Churchill Livingstone.

[11] Madan et al. (2011). Serum Cytokine Profile in Leprosy and Its Correlation WithClinico-Histopathological Profile. Lepr Rev. 82:371-82.

[12] Degang et al. (2014). Leprosy as A Model of Immunity. Future Microbiol. 9 (1): 43-54.

[13] Stefani M, Martelli C. M., Gillis T. P., Kranhenbuhl J. L. (2003). In situ type 1 cytokine gene expression and mechanism associated with early leprosy progression. JID. 188: 1024-1030.

[14] Moubasher, A. E. A., Kamel, N. A., Zedan, H. \& Raheem, D. E. A. (1998a). Cytokines in leprosy. Int J dermatol. 37: 733-40.

[15] Moubasher, A. E. A., Kamel, N. A., Zedan, H. \& Raheem, D. E. A. (1998b). Cytokines in leprosy, II. Effect of treatment on serum cytokines in leprosy. Int J Dermatol. 37: 741-6.

[16] Bandjar.(2015). Analisis Titer Interleukin-12 dan Interleukin-4 Pada Penderita Kusta Multibasiler Pre dan Post Terapi 3 Bulan Dengan Multidrug-Therapy-World Health Organization. Makassar: Program Pascasarjan Universitas Hasanuddin.

[17] Misra N, Murtaza A, Walker B, Narayan N, Misra R. S., Ramesh V. (1995). Cytokine profile of circulating $T$ cells of leprosy patients reflects both indiscriminate and polarized T-helper subsets: T-helper phenotype is stable and uninfluenced by related antigens of Mycobacterium leprae. Immunology. 86: 97-103. 